\title{
Contaminantes en los cuerpos de agua del sur de Sonora
}

\author{
Olga López-Ríos, M.C.(1) Miguel Lechuga-Anaya, M.C.(2)
}

\begin{abstract}
López-Ríos O, Lechuga-Anaya M.
Contaminantes en los cuerpos

de agua del sur de Sonora.

Salud Publica Mex 2001;43:298-305.

El texto completo en inglés de este artículo está disponible en: http://www.insp.mx/salud/index.html
\end{abstract}

\section{Resumen}

Objetivo. Ahondar en el conocimiento sobre la incidencia de las actividades productivas del hombre en su medio, $y$ el riesgo potencial que representa el deterioro ambiental para su propia salud y la sobrevivencia de especies animales. Material y métodos. Se utilizó el paquete informático Decision Support System for Industrial Pollution Control DSS (IPC), desarrollado por el Banco Mundial, la Organización Panamericana de la Salud (OPS) y la Organización Mundial de la Salud (OMS), que se aplicó a un estudio de caso sobre los cuerpos de agua del sur de Sonora, durante 1996. Resultados. Estos sugieren que existen al menos tres factores que han contribuido a la desaparición de criaderos naturales de pescado y camarón en el municipio de Huatabampo: a) la filtración de fertilizantes y pesticidas en las aguas de los drenes; b) el asolvamiento producido por el depósito continuo, en el pasado, de agua de cola de la industria, c) y la descarga inapropiada de aguas negras en los drenes. El texto completo en inglés de este artículo está disponible en: http://www.insp.mx/salud/index.html

Palabras clave: contaminación ambiental; drenaje de agua; recursos hídricos; México

\author{
López-Ríos O, Lechuga-Anaya M. \\ Population, environment and health: pollution \\ in water bodies in the South of Sonora. \\ Salud Publica Mex 2001;43:298-305. \\ The English version of this paper \\ is available at: http://www.insp.mx/salud/index.html
}

\section{Abstract}

Objective. To contribute to the knowledge on the impact of human productive activities on the environment, human health, and the survival of animal species. Material and Methods. A study case of water bodies in the south of Sonora was conducted in 1996. The software Decision Support System for Industrial Pollution Control DSS-IPC (IPC)IPC developed by the World Bank and the Pan American Health Organization was used to analyze data. Results. At least three factors have contributed to the loss of fish and shrimp hatcheries in the municipality of Huatabampo: the filtration of fertilizers and pesticides into the irrigation drainage system, blockage produced by the continuous disposal of industrial waste water, and the discharge of sewer water into the drainage system. Conclusions. Study findings provide evidence that environmental deterioration resulting from human activity is decimating the population of fish and shrimp in the area.The English version of this paper is available at: http://www.insp.mx/salud/index.html

Key words: environmental pollution; drainage, sanitary; water resources; Mexico

(I) Instituto Nacional de Salud Pública, México.

(2) Instituto Tecnológico de Estudios Superiores de Monterrey, México

Fecha de recibido: 13 de julio de 1999 - Fecha de aprobado: 9 de noviembre de 1999

Solicitud de sobretiros: Dra. Olga López Ríos, Departamento de Ingeniería Industrial y de Sistemas, Instituto Tecnológico de Estudios Superiores de Monterrey, Campus Ciudad de México, Calle del Puente 222 colonia Ex-ejidos de Huipulco,Tlalpan, I4380 México D.F., México.

Correo electrónico: ollopez@campus.ccm.itesm.mx 
E 1 agudo deterioro de los ecosistemas impone a la sociedad adoptar no sólo medidas de reordenamiento ecológico, sino también hacer estudios que permitan comprender en qué medida y cuáles actividades del hombre contribuyen a tal deterioro y que, al mismo tiempo, ayuden a evaluar si está en peligro la salud del hombre. En un sentido estricto, el volumen de satisfactores que se pueden producir en una región determinada puede verse disminuido en el tiempo como consecuencia del agotamiento de los recursos fuente de la producción. Este agotamiento puede ser producto de dos factores principales o de una combinación de ambos: a) el abuso de los recursos en respuesta a las formas de una cierta racionalidad de rentabilidad, o b) el agotamiento de los recursos como producto de una explotación inapropiada. ${ }^{1}$

Extender el esquema de la relación entre población y medio ambiente y salud, requiere de una atención particular, porque existen factores de tiempo que pueden ser muy importantes entre el impacto de un acto contaminante y su repercusión en la salud del hombre. Para ahondar en el conocimiento de esta relación es necesario utilizar herramientas nuevas que brinden un conocimiento cuantitativo más exacto del impacto de una actividad productiva sobre el medio, que tome en cuenta la presión poblacional, las características del medio y las normas de salud establecidas. A este respecto el paquete Decision Support System for Industrial Pollution Control (DSS-IPC System), ${ }^{*}$ del Banco Mundial, que fue desarrollado como una herramienta para evaluar la calidad de aire, desechos de agua y desechos sólidos producidos por industrias fijas y unidades móviles en zonas industriales, permite conocer de dónde provienen los principales contaminantes que contribuyen al deterioro ambiental o de recursos, y también del riesgo potencial que se puede tener, a corto o mediano plazo, por una actividad específica sobre un ecosistema, una actividad productiva y sobre la salud del hombre.

Técnicamente, el IPC es un sistema que se origina del estudio y parámetros del documento publicado en 1989 por la Organización Mundial de la Salud sobre control y administración del medio ambiente. ${ }^{2}$ Dicho brevemente, es un paquete que comprende un conjunto muy extenso de bases de datos y módulos de cálculo, referentes a descargas estándar de una gran variedad de actividades económicas e industriales por

\footnotetext{
* Una copia del software que contiene un manual de uso se puede obtener en la siguiente dirección: http:/ / www.unescap.org/stat/ envstat/env-link3.htm
}

sector. Los módulos de cálculo permiten obtener estimaciones fiables de concentraciones contaminantes debidas a actividades económicas en una localidad específica, costos totales de control y costos marginales en el largo plazo, para alcanzar un cierto nivel de reducción de contaminantes. Una descripción más exhaustiva de sus alcances se puede ver en el "help" del software, que se puede obtener en el sitio web antes citado.

El presente estudio se puede enmarcar dentro de un conjunto de aplicaciones del IPC en el territorio mexicano, que tiene como precedente sólo dos ensayos, el primero en la región de Coatzacoalcos-Minatitlán, ${ }^{3}$ y el segundo en la ciudad de Tijuana. ${ }^{*}$

El objetivo principal de este trabajo, es mostrar que el tipo de estudios de carácter técnico en los que el IPC se agrupa son indispensables en todo estudio global de población y medio ambiente y salud, pues pone de manifiesto la incidencia de las actividades del hombre sobre su medio, además de aportar directamente un conocimiento del impacto, cuantificado, del deterioro ambiental por la concentración de contaminantes que pueden incidir negativamente sobre la población, en materia de salud, y un conocimiento indirecto del impacto de la contaminación sobre actividades productivas específicas. El trabajo se centró en los cuerpos de agua (drenes) del sur de Sonora. Por otra parte, este estudio deberá contribuir al esfuerzo de adaptar el paquete IPC, que se ha mostrado de amplia utilidad en países desarrollados, a las condiciones y necesidades de un país como México, poniendo en relieve su potencialidad en dominios afines al industrial (como el agroindustrial), considerando sus limitaciones de aplicación directa debido a la falta de información apropiada disponible.

$\mathrm{Su}$ aplicación se efectuó en lo que denominamos la región Sur de Sonora, que está compuesta por los municipios de Huatabampo, Alamos, Navojoa, Etchojoa, Quiriego, Bacum, Ciudad Obregón y Rosario. En ellos se localizan los principales distritos de riego del estado, éstos son alimentados por los ríos Yaqui y Mayo a través de diez drenes: Cero, Mocarit, Riito, Jupateco, Dátil, Margen Izquierdo, Colector 1, Esperancita, Calle 300 y Principal Bajo.

Después de una visita a la región, en la cual se entrevistó a investigadores de diferentes ramas (preferentemente la agrícola) y autoridades municipales,

\footnotetext{
* Organización Panamericana de la Salud. Tijuana, México: Identificación de zonas de riesgo por contaminantes y giro industrial. Reporte versión preliminar, 1996.
} 
se efectuó una primera evaluación de los principales problemas que afectan a la región.* Al mismo tiempo, se recolectó información de estudios afines, así como la información básica necesaria para la aplicación del IPC.

Los principales problemas que presenta la región son:

- Un deterioro importante de la calidad de la tierra, que incide directamente sobre la producción agrícola y la calidad del agua de los mantos acuíferos.

- Una contaminación periódica del aire, muy importante, por la quema de la gavilla, paja remanente de la cosecha de trigo, que en grandes volúmenes se desecha de esta forma.

- Una contaminación extrema de los drenes por el desecho en ellos de aguas no tratadas.

- Una disminución importante (y desaparición en algunos casos) en la actividad pesquera.

En sí, el problema más extenso es el relacionado con las actividades agrícolas; $;$ el uso intensivo e inadecuado de fertilizantes y pesticidas ha hecho que la tierra se agote o tenga problemas de salinización. En muchos casos directamente, o por filtración, los drenes de riego reciben cantidades importantes de fertilizantes y pesticidas, además de aguas negras en exceso, lo que agrava la situación agrícola pues el agua destinada al riego presenta un alto contenido de coliformes, convirtiéndola en impropia para su destino primario. Así, los drenes son, además del suelo, el principal medio donde se captan los contaminantes generados en el sur del estado de Sonora. Mediciones del Centro de Investigaciones Agropecuarias del Noreste (Ciano), muestran que éstos, al descargarse al mar en sus bahías, acaban con especies marinas vegetales y animales, afectando notablemente la actividad de cultivo del camarón. Esto lo corrobora la opinión de los cooperativistas pesqueros, que asocian la baja en la actividad pesquera de manera directa con las descargas de agua hacia el mar, que han incidido de manera importante, según su experiencia a través del tiempo, en la reproducción de especies marinas. Por ejemplo, la desembocadura del Dren Mocarit era hasta hace poco tiempo un lugar de reproducción natural

\footnotetext{
* Presidencia Municipal de Huatabampo. Presentación del C. Presidente Municipal de Huatabampo de la problemática ambiental del Municipio. Huatabampo, Sonora, México, 1995.

‡ Núñez A. Fertilización nitrogenada y fosfórica del trigo en la zona Fuerte-Mayo. México, D.F.: Centro Regional Unidad Noroeste (CRUNO), 1995. Documento no publicado.
}

de pescado y camarón; actualmente la alta contaminación por aguas negras y un crecimiento desmedido del manglar han hecho desaparecer prácticamente este criadero.

La información utilizada para la aplicación del IPC proviene de un trabajo de campo de colección de la misma mediante encuestas, y a partir de entrevistas locales en las diferentes industrias, de los reportes periódicos de la Comisión Nacional del Agua (CNA), del Centro Regional Unidad Noroeste (Cruno) de la Universidad Autónoma de Chapingo y del Ciano. La información es actual y se puede considerar fiable pero por desgracia, en algunas variables es incompleta. Por ejemplo, los datos de la calidad del agua provienen de un mismo punto de monitoreo y no son regulares, pero es suficiente para tener un primer acercamiento a la situación en la región. Los resultados obtenidos se deben considerar como parciales y limitados en espacio y tiempo. Ellos dan un espectro mínimo de la presencia de contaminantes. Por las características de la información que requiere el IPC, ésta no está disponible bajo el esquema de una publicación, pues son mediciones en general, por lo que para el presente estudio referimos al lector interesado a los autores, y para un estudio similar, a las fuentes directas de las instituciones mencionadas.

En lo que respecta a la quema de la gavilla, que constituye un problema muy grave en la región, a juicio de investigadores tanto del Cruno como del Ciano, su estudio requiere de mediciones de la calidad del aire, las cuales son inexistentes por la falta de interés de las autoridades correspondientes. Estas se podrían obtener mediante la instalación de aparatos en zonas estratégicas de la región. La simple observación muestra que en épocas de quema el ambiente se vuelve irrespirable, con capas de humo que semejan una densa niebla a lo largo de cientos de hectáreas. La industria es esencialmente de conservación de alimentos y producción de fertilizantes y pesticidas, y se carece de estadísticas sobre desechos y emisiones al aire por ella generados.

La limitada disponibilidad de información o lo escaso y poco confiable de alguna parte de ella, así como los problemas más latentes relacionados con la aplicación del paquete, redujeron el presente estudio a los cuerpos de agua formados por los diez drenes abastecidos por los ríos Yaqui y Mayo.

Como resultado del levantamiento de información local se obtuvo la siguiente: la actividad industrial en el Sur de Sonora es esencialmente agrícola y de procesamiento de sardina. Los procesos principales son el enlatado, la producción de harina de pescado y la producción de fertilizantes y pesticidas.

Entre las principales empresas se encuentran: 
- Grupo Industrial Mayo. Este grupo está ubicado en el municipio de Huatabampo. Sus aguas residuales son depositadas en el Dren T-O, el más cercano al mar, inactivo para el riego y que funciona como laguna de oxidación. Las medidas de tratamiento de esas aguas son inadecuadas y afectan a las comunidades circunvecinas, por los malos olores que de ahí se despiden y el acarreo de bacterias patógenas por los vientos.

- Yávaros Industrial. Esta es una de las empresas más importantes en el ramo de procesamiento de sardina, pues produce 700 mil cajas, de 48 latas, anualmente. En el pasado esta industria depositaba toda su agua de cola y desechos de sardina en la bahía de Yávaros, lo que explica el asolvamiento y la grave contaminación existentes. Actualmente, las autoridades municipales han ejercido un control de la situación y desde 1995 los desechos se depositan en una laguna de oxidación.

- Tezal, Productos Pesqueros del Golfo e Industrias Barda. Estas son industrias productoras de harina de pescado y corte de sardina que operan en la temporada de pesca de esta última. Al igual que Yávaros Industrial, depositan desde 1995 sus aguas residuales en una laguna de oxidación.

- Agro-industrias Mayo. Esta empresa produce 30 mil toneladas de fertilizante para uso de la región y estados vecinos. Sus desechos van sin tratamiento al Dren las Animas.

- Industrias Agrícolas del Sur de Sonora. Dicha corporación produce 40 mil toneladas de aguamonia y amoniaco, y 80 mil toneladas de insecticidas en polvo, destinados a la actividad agrícola.

Las cantidades, por grandes rubros de producción, se observan en el cuadro I, el cual muestra que grandes giros de producción que generan desechos de materia orgánica son los de enlatado de vegetales y sardina.

\section{Material y métodos}

El paquete IPC utiliza siete modelos de dispersión y está basado en información de producción industrial, análisis de laboratorio, características de suelo, aire, agua y de condiciones meteorológicas y geográficas específicas que imperan en la región de estudio. Este paquete compara las concentraciones observadas en el medio de estudio con las normas internacionales de salud establecidas por la Organización Mundial de la Salud e identifica medidas viables de control. Con su

\begin{tabular}{|c|c|c|}
\hline \multicolumn{3}{|c|}{$\begin{array}{l}\text { PRODUCCIÓN EN EL SUR de SONORA } \\
\text { POR GIRO y PROCESO. MÉXICO, I } 996\end{array}$} \\
\hline Giro & Proceso & $\begin{array}{l}\text { Unidades de producción } \\
\text { (I } 000 \text { unidades) }\end{array}$ \\
\hline Preservación de frutas & Vegetales deshidratados & $15120000.00 \mathrm{t}$ \\
\hline y vegetales & Chiles & $320000.00 \mathrm{t}$ \\
\hline Sardina & Enlatado & $30800000.00 \mathrm{t}$ \\
\hline Manufactura de & Nitrato de amonia & $168.00 \mathrm{t}$ \\
\hline \multirow[t]{4}{*}{ fertilizantes y pesticidas } & Sulfato de amonia & $1667.70 \mathrm{t}$ \\
\hline & Urea & $258.00 \mathrm{t}$ \\
\hline & Acido fosfórico & $419.60 \mathrm{t}$ \\
\hline & Fosfatos & $1064.50 \mathrm{t}$ \\
\hline
\end{tabular}

módulo de costos permite el cálculo del valor de las medidas estratégicas de control, lo que permite evaluar en parte el costo ecológico de una acción o actividad específica.

La aplicación del paquete IPC se efectuó con base en la información recolectada sobre la producción de las principales actividades agroindustriales, del procesamiento de productos del mar y de los análisis químicos de calidad del agua provenientes de los distintos puntos de monitoreo que reporta la CNA. Se utilizaron valores promedio, se supuso implícitamente que los procesos incorporados en el modelo son los únicos que contribuyen a la contaminación de los drenes y que ésta no proviene de otra parte. En este punto hay que resaltar que las concentraciones establecidas por el IPC son el nivel mínimo del espectro de contaminantes presentes. Se sabe que los pesticidas contribuyen de manera importante a la contaminación de los drenes, lo mismo que los desechos de aguas negras, y de los cuales no disponemos datos de descarga.

A continuación describimos las cargas máximas de contaminantes generadas por giro y proceso de producción.

\section{Resultados}

\section{Inventario de cargas contaminantes por giro y proceso}

El cuadro II retoma los principales contaminantes presentes en la región. En él se indica qué proceso y qué giro contribuyen de manera importante con algún con- 
taminante específico. El contenido del cuadro es muy importante, pues permite identificar el proceso que está generando la contaminación.

En el cuadro II se observa también que la demanda bioquímica de oxígeno $\left(\mathrm{DBO}_{5}\right)$ proviene esencialmente del proceso de enlatado, el cual genera los sólidos suspendidos y el aceite. El resto de los contaminantes provienen de la producción de fertilizantes y pesticidas en los que no hay materia orgánica.

Una de las características importantes del IPC es que contiene un listado de las concentraciones de compuestos químicos toleradas por el hombre, que son comparadas con las concentraciones de compuestos químicos detectadas en los cuerpos de agua o el medio ambiente.

\section{Concentración ambiental de contaminantes}

El reporte de concentración de contaminantes en los cuerpos de agua muestra que, en términos generales, en los diez drenes las concentraciones exigidas por las normas internacionales de salud están por encima de las cantidades detectadas, salvo para el fluoruro en el dren Riito en el cual se superan las normas establecidas. Se presenta en el cuadro III en detalle el reporte para el dren el Dátil, análogo para los otros.

Otra de las particularidades importantes del IPC, es que permite conocer los contaminantes que se emiten de acuerdo con los procesos productivos existentes, ${ }^{*}$ independientemente que se introduzcan datos de descarga. Por este motivo, en el caso del arsénico, el valor representa un valor mínimo del espectro de contaminante presente, pues los análisis de calidad de agua no aportan ninguna información al respecto por falta de reactivos para los análisis respectivos. Dado que el valor calculado por el IPC se encuentra entre 0.5-1 (que resulta del cociente de la concentración que marca la norma internacional de salud), que si bien indica que se respetan las normas, sería prudente ahondar en el estudio de la presencia de este contaminante, por los riesgos que representa para la salud del hombre y para las especies de flora y fauna acuática que se reproducen en la desembocadura de algunos drenes.

Otro de los principales problemas que presentan los drenes es el alto contenido de coliformes, generado por el desecho en ellos de aguas negras. La importancia de estas cantidades de coliformes por cada 100 $\mathrm{ml}$, se debe a su capacidad para convertir el agua en

\footnotetext{
* El paquete contiene una gran cantidad de procesos industriales que se seleccionan obviamente en función de aquellos presentes en la región; el tipo de errores que provienen de esta elección pueden surgir de que se partió del supuesto de una cierta organización del proceso industrial, que no necesariamente puede ser el mismo de la región. Sin embargo recalquemos que el espectro de presencia de contaminantes parte del mínimo, por lo que de aparecer algún problema seguramente este es más agudo, en caso contrario, de existir sospecha, se deberá aumentar el número de mediciones.
}

Cuadro II

Carga máxima de contaminante por giro y proceso. Sonora, México, 1996

Contaminante

Giro

Fertilizantes

Arsénico

Demanda bioquímica de oxígeno $\mathrm{DBO}_{5}$

Cadmio

Cromo

Cobre

Fluoruro

Mercurio

Nitrógeno de amonia

Níquel

Nitrato de amonia

Grasas y aceite

Plomo

Fósforo

Sólidos suspendidos

Zinc
Enlatado de sardina y de legumbres

Fertilizantes

Fertilizantes

Fertilizantes

Fertilizantes

Fertilizantes

Fertilizantes

Fertilizantes

Fertilizantes

Enlatado de sardina

Fertilizantes

Fertilizantes

Enlatado de sardina

Fertilizantes
Proceso

Carga (t/año)

Producción de ácido fosfórico

2.52

Preservación de alimentos $\quad 442512 \quad 170.00$

Producción de ácido fosfórico

Producción de ácido fosfórico

12.75

Producción de ácido fosfórico

12.98

Producción de ácido fosfórico

114099.00

Producción de ácido fosfórico

0.42

Producción de ácido fosfórico

27.20

Producción de ácido fosfórico

Producción de ácido fosfórico

2516.00

Preservación de Alimentos

52360000.00

Producción de ácido fosfórico

Producción de ácido fosfórico

$253557 \mid 51.00$

Preservación de alimentos

Producción de ácido fosfórico

3.64 
impropia para el consumo animal e incluso para riego. El cuadro IV retoma las cantidades promedio para cada uno de los drenes.

En el modelo no incluimos cargas de desecho de aguas negras. Estos valores provienen del análisis de la calidad del agua efectuados por la CNA en los puntos de monitoreo.

\section{Cuadro III}

Concentración ambiental de Contaminantes: COMPARACIÓN CON LA Guía dE SALUd para el Dren el Dátil. Sonora, México, 1996

\begin{tabular}{|c|c|c|c|}
\hline Contaminante & $\begin{array}{c}\text { Concentración } \\
\text { anual } \\
\text { (mg/l) }\end{array}$ & $\begin{array}{l}\text { Concentración } \\
\text { de la Guía } \\
\text { de salud }\end{array}$ & $\begin{array}{l}\text { \% respecto } \\
\text { a la Guía } \\
\text { de salud }\end{array}$ \\
\hline Arsénico & 0.00001 & 0.00002 & entre 0.5 y I \\
\hline DBOS & 921.53 & & \\
\hline Cadmio & 0.00001 & 0.003 & $<0.5$ \\
\hline Cromo & 0.00003 & 0.12 & $<0.5$ \\
\hline Cobre & 0.00003 & 2.0 & $<0.5$ \\
\hline Fluoruro & $0.64|2|$ & 1.5 & $<0.5$ \\
\hline Mercurio & 0 & 0.001 & $<0.5$ \\
\hline Nitrógeno de amonia & 21.67006 & & \\
\hline Níquel & 0.00003 & 0.02 & $<0.5$ \\
\hline Nitrato de amonia & 0.10532 & & \\
\hline Aceite & 126.788 & & \\
\hline Plomo & 0.00003 & 0.01 & $<0.5$ \\
\hline Fósforo & 0.01595 & & \\
\hline Sólidos suspendidos & 642.01 & & \\
\hline Zinc & 0.00001 & 5.0 & $<0.5$ \\
\hline
\end{tabular}

\section{Cuadro IV \\ Concentración PROMEdio de Coliformes, Sonora, México, 1996}

\begin{tabular}{lc} 
Dren & Coliformes por $100 \mathrm{ml}$. \\
El Dátil & 9383 \\
\hline Calle 300 & 3400 \\
\hline Yupateco & 2300 \\
\hline Cero & 1100 \\
\hline Riito & 900 \\
\hline Canal principal margen izquierdo & 930 \\
\hline Mocarit & 2300 \\
\hline Colector I & 4640 \\
\hline Canal principal bajo & 4600 \\
\hline Esperancita & 4680
\end{tabular}

\section{Discusión}

Con base en los contaminantes listados anteriormente, se elaboró para cada uno de ellos el costo de las medidas de control que eliminarían a largo plazo el contaminante presente en el cuerpo de agua. Estos representan el costo promedio marginal a largo plazo, basado en los valores y costos integrados dentro del paquete IPC, que corresponden a costos promedio para los Estados Unidos de América, (figura 1), que retoma algunos de los costos ligados a limpiar los drenes de los contaminantes presentes. Si se supone que el material $^{*}$ de depuración se debe importar, se puede considerar que estos costos son costos reales para el país, pues el equipo de medio ambiente no causa impuestos importantes. Los costos de mano de obra y energía están calculados con base en los costos de la energía en la región y con base en un costo de mano de obra de 6 USD $\$$ /hora; este supuesto del costo de la mano de obra podría no ser el más óptimo, pero se aproxima al costo de emplear personal especializado. Por otra parte, hay que considerar que se trata de costos mínimos, pues en el modelo no se han incluido datos de descarga de aguas negras, pesticidas y otras posibles descargas relacionadas con las actividades porcícolas y avícolas.

En la figura 1 observamos que los costos marginales son relativamente bajos en la primera etapa del proceso y posteriormente crecientes, esto es válido para los contaminantes: arsénico, nitrógeno de amonia, demanda bioquímica de oxígeno, aceite, partículas suspendidas, cromo, cadmio, cobre, fluoruro, mercurio, níquel, plomo, compuestos de fósforo y zinc.

De acuerdo con los resultados obtenidos en la ejecución del paquete IPC, se aprecia que los compuestos que intervienen en la elaboración de fertilizantes aparecen también en los compuestos encontrados en los análisis de la calidad del agua en los distintos drenes. Si bien su concentración no es importante de acuerdo con las normas internacionales de salud, los resultados no pueden considerarse concluyentes, pues los datos de monitoreo son parciales, proviniendo siempre de los mismos puntos, pues no son exhaustivos en el tiempo, y en algunos casos cubren sólo un periodo de cuatro meses.

\footnotetext{
* El balance de los costos marginales, para limpiar de un contaminante específico los cuerpos de agua en estudio, es de suma importancia, pues sirve de evaluación monetaria del costo ecológico de las actividades productivas del hombre que inciden de diversas maneras sobre el ambiente y los recursos.

‡ Dólares estadunidenses.
} 

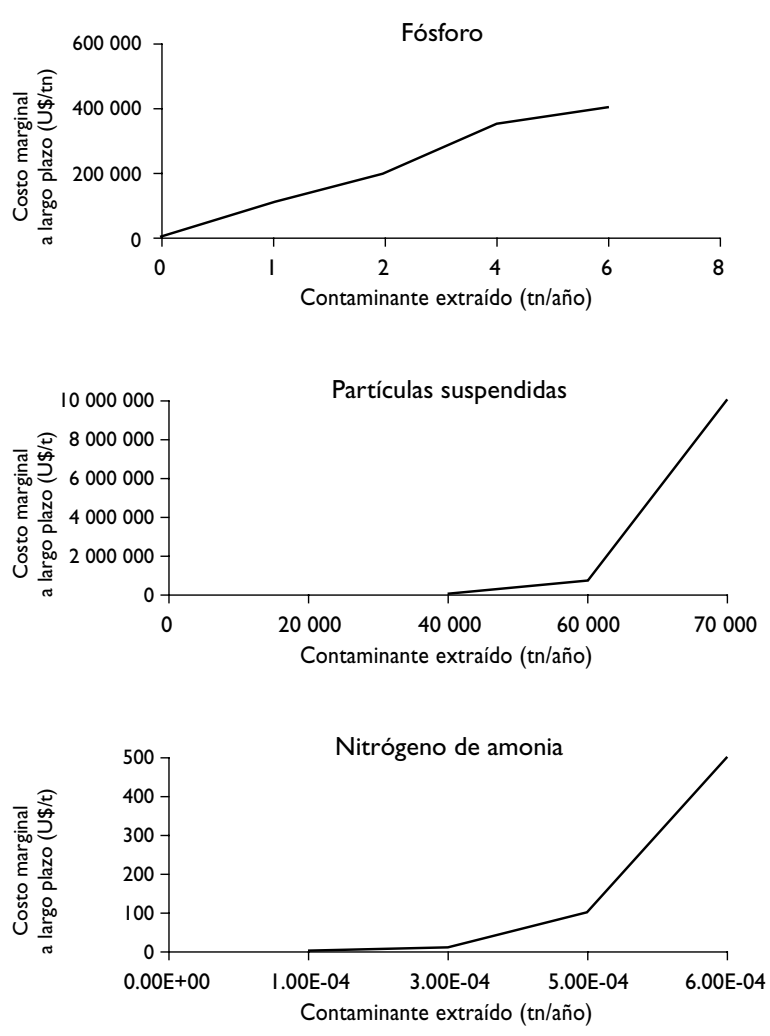
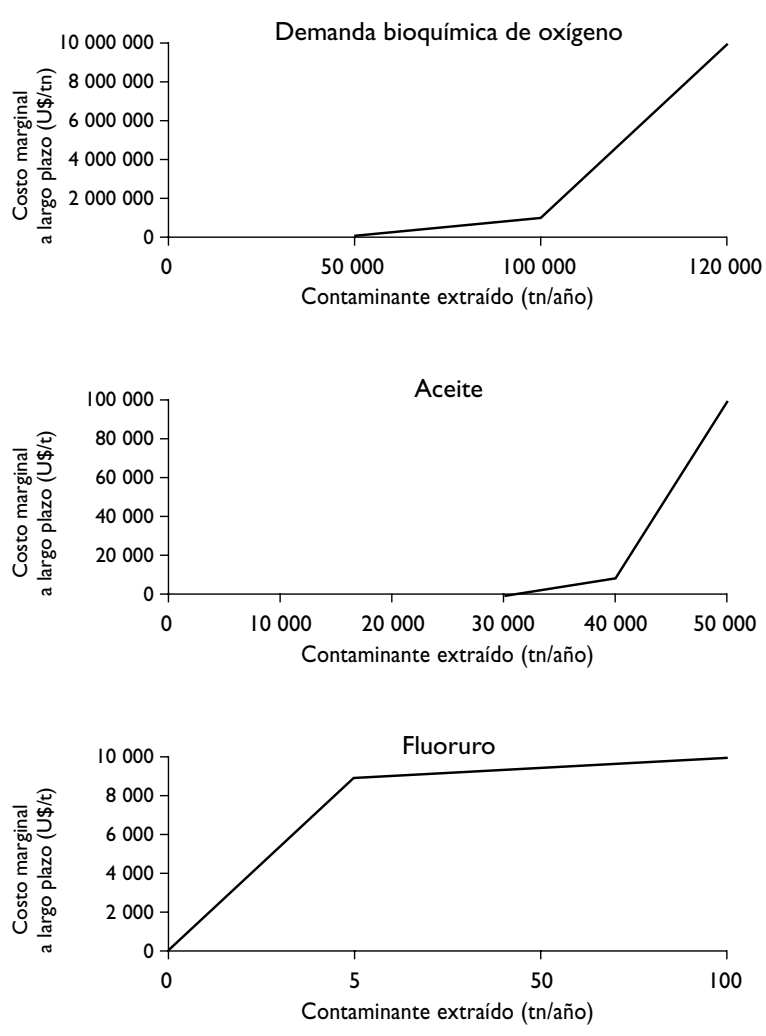

Figura I. Costo marginal PROMEdio de EXtracción de CONTAMINANTES a LaRgo plazo

Un resultado inquietante es la cantidad de coliformes presentes por cada $100 \mathrm{ml}$ en los diez drenes. Si bien podemos aceptar que el resultado no es concluyente para calificar el agua de impropia para riego (su destino primario), pues depende del punto de monitoreo, si nos muestra un problema extremo de contaminación en esos puntos, y podríamos concluir que de esos puntos en adelante esa agua no se debería usar para actividades de riego, debido principalmente a las características de los drenes y a la ausencia total de plantas tratadoras de agua. Por otra parte, la demanda importante de oxígeno y los sólidos suspendidos presentes, constituyen también un problema importante.

Las concentraciones de contaminantes observadas muestran valores relativamente bajos. Esto se puede explicar por la poca cantidad de industrias y giros industriales que existen en la región. Pero a pesar de que las concentraciones son bajas, es probable que aquéllos se hayan ido acumulando con el tiempo, principalmente en el lugar de la descarga de los drenes, particularmente en la salida del dren Moncarit, lo que explicaría el crecimiento acelerado del manglar que ahí se encuentra. Otro aspecto importante para profundizar es que algunos contaminantes generados por los fertilizantes, como metales y arsénico, pueden ser los causantes de la extinción de la fauna marina en la descarga del dren Moncarit en el municipio de Huatabampo.

A este respecto, la contaminación en Huatabampo no puede atribuirse exclusivamente a la industria, pues es relativamente escasa. El problema es esencialmente de acumulación, pues el dren Moncarit recibe por filtración, fertilizantes y pesticidas, además de aguas de drenaje de los municipios que se encuentran al norte de Huatabampo. Es urgente la instalación de plantas tratadoras de agua en este dren y proceder a un control estricto de las aguas que en él se desechan, pues se han deteriorado de manera casi total las actividades camaronera y reproductiva de flora y fauna 
acuática cercana al litoral. El costo beneficio de tales acciones es sin duda positivo por la importancia de esta actividad económica.

Es necesario profundizar en rubros de interés detectados en esta primera aproximación analítica sobre la situación real de los cuerpos de agua, del suelo y del aire. Se requieren estadísticas y monitoreos regulares de la calidad del aire y del agua. Los resultados obtenidos para los distintos drenes muestran que se precisa de más puntos de monitoreo, y de una regularidad en las mediciones, para que los resultados no sean parciales. Para una aplicación completa del IPC se requiere de encuestas sobre cantidades de desechos sólidos generados, de monitoreos de la calidad del aire a lo largo del año, principalmente en los meses de la quema de la gavilla, que permitan evaluar la gravedad de la situación.

Teniendo en cuenta que la actividad principal de la región es la agricultura, es urgente realizar un estudio evaluatorio total en ésta de la potencialidad o degradación de la tierra, del grado de contaminación de los mantos acuíferos y del litoral. Un estudio de esta envergadura se debe llevar a cabo durante varios meses, pues el peligro en que se encuentran actividades generadoras de riqueza para la región y para el país lo justifican.

Finalmente, retomando el objetivo planteado, se puede concluir que, efectivamente, los resultados obtenidos, mediante un ejercicio técnico como el presente ensayo, son de gran importancia dentro del análisis de las relaciones población y medio ambiente, ya que ponen de manifiesto que el desarrollo incontrolado de las actividades generadoras de riqueza puede provocar la desaparición de otras e incluso, a largo plazo, la de la misma actividad. Se puede decir que la incidencia de un medio ambiente contaminado sobre la salud del hombre, puede relacionarse con los índices de contaminación detectados por contaminante específico, y que mediante el análisis de costos de eliminación de un contaminante se puede establecer el costo ambiental de una actividad económica específica.

Este espectro de resultados es un conjunto de información mínima y muestra que el paquete IPC tiene un gran uso potencial en el país, y que efectivamente se requiere del levantamiento de encuestas muy específicas para una aplicación completa y adecuada del mismo, pues los resultados obtenidos con él retribuyen ampliamente el costo en el que se incurra al aplicarlo.

\section{Referencias}

I. Toledo M, Carabias J, Toledo C, Gónzalez P. La producción rural en México: alternativas ecológicas. México, D.F. Fundación Universo Veintiuno, 1989.

2. World Health Organization. Management and Control of the Environment. Working document. Ginebra:WHO, 1989.

3. Banco Mundial. Application of the decision support system (DSS) for industrial pollution control (IPC) to the regions of Coatzacoalcos y Minatitlán. The World Bank, Environment Department. Environmental Economics and Pollution Division. Washington D.C.:World Bank 1994. 